\title{
Fully quantitative cardiovascular magnetic resonance myocardial perfusion ready for clinical use: a comparison between cardiovascular magnetic resonance imaging and positron emission tomography
}

Henrik Engblom¹, Hui Xue², Shahnaz Akil ${ }^{1}$, Marcus Carlsson ${ }^{1}$, Cecilia Hindorf ${ }^{3}$, Jenny Oddstig ${ }^{3}$, Fredrik Hedeer ${ }^{1}$, Michael S. Hansen², Anthony H. Aletras ${ }^{1,4}$, Peter Kellman² and Håkan Arheden ${ }^{1 *}$

\begin{abstract}
Background: Recent studies have shown that quantification of myocardial perfusion (MP) at stress and myocardial perfusion reserve (MPR) offer additional diagnostic and prognostic information compared to qualitative and semi-quantitative assessment of myocardial perfusion distribution in patients with coronary artery disease (CAD). Technical advancements have enabled fully automatic quantification of MP using cardiovascular magnetic resonance (CMR) to be performed in-line in a clinical workflow. The aim of this study was to validate the use of the automated CMR perfusion mapping technique for quantification of MP using $13 \mathrm{~N}-\mathrm{NH} 3$ cardiac positron emission tomography (PET) as the reference method.

Methods: Twenty-one patients with stable CAD were included in the study. All patients underwent adenosine stress and rest perfusion imaging with $13 \mathrm{~N}-\mathrm{NH} 3 \mathrm{PET}$ and a dual sequence, single contrast bolus CMR on the same day. Global and regional MP were quantified both at stress and rest using PET and CMR.

Results: There was good agreement between global MP quantified by PET and CMR both at stress $(-0.1 \pm 0.5 \mathrm{ml} / \mathrm{min} / \mathrm{g})$ and at rest $(0 \pm 0.2 \mathrm{ml} / \mathrm{min} / \mathrm{g})$ with a strong correlation $(r=0.92, p<0.001 ; y=0.94 x+0.14)$. Furthermore, there was strong correlation between CMR and PET with regards to regional MP $(r=0.83, p<0.001 ; y=0.87 x+0.26)$ with a good agreement $(-0.1 \pm 0.6 \mathrm{ml} / \mathrm{min} / \mathrm{g})$. There was also a significant correlation between CMR and PET with regard to global and regional MPR ( $r=0.69, p=0.001$ and $r=0.57, p<0.001$, respectively).
\end{abstract}

Conclusions: There is good agreement between MP quantified by $13 \mathrm{~N}-\mathrm{NH} 3 \mathrm{PET}$ and dual sequence, single contrast bolus CMR in patients with stable CAD. Thus, CMR is viable in clinical practice for quantification of MP.

Keywords: Myocardial perfusion, Cardiac magnetic resonance, Positron emission tomography, Myocardial perfusion reserve

\footnotetext{
* Correspondence: hakan.arheden@med.lu.se

${ }^{1}$ Department of Clinical Physiology, Clinical Sciences, Lund University and

Lund University Hospital, Lund, Sweden

Full list of author information is available at the end of the article
} 


\section{Background}

Ischemic heart disease (IHD) is the most common cause of mortality and morbidity in the western world. Obstructive coronary artery disease (CAD) compromising myocardial perfusion is the main pathophysiological mechanism underlying stress-induced myocardial ischemia. The extent of stress-induced ischemia due to CAD has been shown to be of important prognostic value [1, 2]. Significant advances in both diagnosing and treating epicardial CAD have improved survival and reduced morbidity during the last decades in patients suffering from this disease [3-5]. Most clinical methods used for diagnosing stress-induced myocardial ischemia focus on epicardial obstructive CAD and rely on qualitative or semi-quantitative assessment of the relative spatial perfusion distribution i.e. myocardial perfusion single photon emission computed tomography (SPECT), first-pass perfusion cardiac magnetic resonance (CMR) and non-dynamic cardiac positron emission tomography (PET). There are, however, data suggesting that the use of qualitative or semi-quantitative methods for assessing stress-induced ischemia have limited ability to depict the full extent of the atherosclerotic coronary disease [6]. Furthermore, recent studies have shown that full quantification of myocardial perfusion (MP) expressed in terms of $\mathrm{ml} / \mathrm{min} / \mathrm{g}$ myocardial tissue offers independent prognostic information in patients with CAD [7-9].

Cardiovascular magnetic resonance (CMR) with a dual sequence, single contrast bolus approach has been shown to enable quantitative assessment of MP [10]. However, there have been challenges associated with this technique such as the non-linear relationship between signal intensity and gadolinium concentration, respiratory motion correction and gadolinium kinetic modelling. Recent technical developments have shown promising results to overcome these challenges [11]. The proposed CMR perfusion mapping method is implemented in-line, is fully automatic and is readily integrated into the clinical workflow via the Gadgetron framework [12]. Thus, this technique seems to be ready for clinical implementation, making quantitative perfusion available as a routine examination in patients with suspected or established ischemic heart disease. Even though this is a promising technique, clinical validation against an independent reference standard is still lacking.

Cardiac positron emission tomography (PET) with dynamic image acquisition during bolus injection of a radioactive perfusion tracer at rest and stress is currently considered to be the clinical reference method for quantitative assessment of MP. However, cardiac PET is of limited use as a wide spread routine clinical examination due to its limited availability and sometimes challenging logistics as well as due to the ionizing radiation associated with this technique.
Therefore, the aim of this study was to validate MP by a fully automatic, in-line, dual sequence, single contrast bolus CMR perfusion mapping sequence using cardiac $13 \mathrm{~N}-\mathrm{NH} 3 \mathrm{PET}$ as the reference method in stable CAD patients.

\section{Methods \\ Study population and study design}

The study protocol was approved by the regional ethical committee and all subjects gave written informed consent prior to participation. Patients eligible for inclusion had all been clinically evaluated and a decision to perform an elective coronary angiography with preparedness for percutaneous coronary intervention (PCI) had been made, with or without prior stress imaging. Patients were excluded if they had contraindications for CMR such as pacemaker or other CMR-incompatible devices or claustrophobia. The patients were recruited between September 2016 and February 2017 and examined at Lund University Hospital, Lund, Sweden. All patients underwent both adenosine stress/rest CMR with dual sequence, single contrast bolus approach as well as adenosine stress/rest $13 \mathrm{~N}-\mathrm{NH} 3$ PET on the same day (4-5 h apart) for assessment of MP. Rate pressure products (heart rate $\mathrm{x}$ systolic blood pressure) were calculated both at rest and stress as a measure of the hemodynamic response to adenosine during PET and CMR, respectively. For 12 patients the CMR and PET examination was performed within a month prior to the angiography. For 9 patients, the CMR and PET examinations were performed as a follow-up approximately 6 months after the angiography.

\section{Dual sequence, single contrast bolus CMR for MP quantification Image acquisition}

All images were acquired on a Magnetom Aera $1.5 \mathrm{~T}$ system (Siemens Healthcare, Erlangen, Germany). A basal, a mid-ventricular and an apical short-axis image were acquired both at rest and at stress. The stress images were acquired during 90 heart beats, starting three minutes after initiation of intravenous adenosine infusion $(140 \mu \mathrm{g} / \mathrm{kg} / \mathrm{min})$. The arterial input function was calculated using the left ventricular (LV) blood pool which was automatically segmented from low resolution images optimized for the high gadolinium concentration. Higher spatial resolution images were used for estimating myocardial perfusion. In order to achieve a linear relationship between the LV blood signal used as an input function and the gadolinium contrast agent concentration a number of steps were taken in the design of the sequence protocol and image reconstruction as previously described [11]. The sequence uses a low flip angle FLASH low resolution protocol with 2 echoes such that the echo times were 
short to minimize $\mathrm{T} 2 *$ losses at high concentration, and so that remaining $\mathrm{T} 2 \%$ losses could be estimated and corrected. The non-linearity of saturation recovery was minimized by using a short saturation delay achieved using a small matrix and parallel imaging to reduce the number of phase encode lines. The remaining non-linear response is corrected by converting to gadolinium concentration units.

Late gadolinium enhancement (LGE) imaging was performed to assess regions of myocardial infarction.

\section{Image reconstruction \& perfusion mapping}

The input function and myocardial images are converted to units of gadolinium contrast agent concentration $(\mathrm{mmol} / \mathrm{L})$ which were input to the tissue model used in quantification [11]. Conversion to gadolinium units effectively addresses the non-linearity between the measured saturation recovery signal and actual gadolinium concentration. Conversion to contrast concentration was accomplished by means of a look-up-table that is calculated by a Bloch simulation of the specific protocol. This look-up-table is recalculated for each scan as part of the image reconstruction. The input to the look-up-table is the measured saturation recovery signal which is normalized by a proton density used as a reference. MP was calculated using a blood tissue exchange model originally developed by Bassingthwaighte [13], which is a distributed model described by partial differential equations. In addition to myocardial blood flow, the model estimates the permeability surface area product for the capillaries which determines the extraction fraction and the intracapillary plasma and interstitial fluid volumes.

\section{Image analysis}

All images were analyzed using the software Segment (v2.0 R5378) [14]. The endo- and epicardial borders for the basal, mid-ventricular and apical short-axis images were manually delineated both at stress and rest. To avoid inclusion of blood pool or extracardiac structures within the regions of interest where MP was quantified, the delineations were kept approximately 1 pixel away from the endo- and epicardial borders. Obvious image artifacts and coronary arteries were excluded from the regions of interest. Myocardial perfusion in $\mathrm{ml} / \mathrm{min} / \mathrm{g}$ was assessed regionally according to the 17-segment model [15], excluding the apical segment, resulting in 16 segments per patient. MP was assessed globally (average MP for all 16 segments). Myocardial perfusion reserve (MPR) was also calculated, defined as the ratio between MP at stress over rest and was expressed both regionally and globally. Both regional and global MP were compared to corresponding measures by PET. Infarct size was assessed from the LGE short-axis images by the use of the recently described EWA (expectation maximization weighted intensity A priori information) algorithm for infarct quantification [16]. In short, the EWA algorithm is based on combining an intensity classification by Expectation Maximization with a pixel intensity weighting approach to account for partial volume effects. Furthermore, a priori information on coronary artery supply territory is taken into consideration for the automatic infarct size quantification.

\section{Cardiac $13 \mathrm{NH}_{3}$-PET for MP quantification Image acquisition and reconstruction}

All patients underwent a standard clinical cardiac $13 \mathrm{~N}-$ NH3 PET at rest and stress, with a minimum of $50 \mathrm{~min}$ between the examinations to allow for physical decay of the radiopharmaceutical. All images were acquired with a Discovery $690 \mathrm{PET} / \mathrm{CT}$ scanner (General Electric Healthcare, Waukesha, Wisconsin, USA). A CT localization image over the chest was acquired to enable accurate patient positioning, followed by a low-dose computed tomography (CT) used for attenuation correction $(120 \mathrm{kV} ; 10 \mathrm{mAs}, 10$; rotation time $0.5 \mathrm{~s})$. Thereafter, the patients received an intravenous injection of $430-600 \mathrm{MBq}$ of $13 \mathrm{~N}-\mathrm{NH} 3$ at rest. An ECG-gated PET acquisition with a total duration of $15 \mathrm{~min}$ was started simultaneously with the tracer injection. For the stress image acquisition, the patient was injected with the same amount of $13 \mathrm{~N}-\mathrm{NH} 3$ after 3 min of adenosine infusion $(140 \mu \mathrm{g} / \mathrm{kg} / \mathrm{min})$ which continued, with continuous ECG monitoring, for $4 \mathrm{~min}$ after the injection of the radiopharmaceutical during which the images for quantitative perfusion were acquired.

The 0-4 min PET acquisition data were reconstructed into dynamic images, with 21 time frames $(12 \times 5 \mathrm{~s}, 4 \times 10 \mathrm{~s}$, $4 \times 20 \mathrm{~s}$ and $1 \times 60 \mathrm{~s})$. The images were reconstructed according to recommendations from the vendor, with OSEM without time-of-flight (TOF), and with point spread function (PSF) modelling, with 3 iterations, 12 subsets and a $5 \mathrm{~mm}$ post filter. Static images were reconstructed from the data acquired 4-15 min after start of injection to depict the relative spatial myocardial perfusion using the following reconstruction parameters: OSEM with TOF and PSF modelling with 5 iterations and 18 subsets and a $3 \mathrm{~mm}$ post filter. The PET and the CT images were checked for patient motion before reconstruction.

\section{Analysis of the quantitative myocardial perfusion}

All PET images were analyzed using the software Carimas (version 2.7, Turku, Finland), developed for quantitative perfusion assessment by cardiac PET, including $13 \mathrm{~N}-\mathrm{NH} 3[17,18]$. The LV endo-and epicardial borders were automatically segmented with manual adjustment when needed. The arterial input function was derived from a blood pool volume of interest which was placed in the basal part of the LV cavity. The activity in the blood 
and the myocardial wall as a function of time served as input function for the deGrado compartment model for $13 \mathrm{~N}-\mathrm{NH} 3$ [19] implemented in the Carimas correcting the input function for extraction fraction of $13 \mathrm{~N}-\mathrm{NH} 3$ (approximately 90\%). Myocardial perfusion was calculated at rest and stress, both globally and in each of the corresponding 16 segments of the left ventricle used for CMR.

\section{Statistics}

Data are expressed as mean \pm standard deviation (SD). The relationship between MP by PET and CMR, both global and regional, as well as MPR was assessed with Pearson's correlation coefficient. The agreement between the MP by PET and CMR was expressed as bias \pm SD and graphically shown as difference plots according to Bland and Altman [20]. The difference in rate pressure products between PET and CMR was assessed by Student's t-test. All statistical analyses were performed using Excel 2013 (Microsoft Corporation, Redmond, Washington, USA). Results with a $p$ value $<0.05$ was considered to be statistically significant.

\section{Results}

Patient characteristics are shown in Table 1. Typical images obtained by PET and CMR in a patient with right coronary artery (RCA) disease and stress-induced ischemia in the inferior LV wall are shown in Fig. 1. Myocardial infarction was detected in 4 of 21 patients. Infarct size in these 4 patients was $8 \pm 5 \%$. Of the 12 patients that underwent CMR and PET before angiography, 4 patients had signs of stenosis in left anterior descending

Table 1 Patient characteristics

\begin{tabular}{ll}
\hline Age (years) & $68 \pm 9$ \\
Female & $4(19 \%)$ \\
Smoking & $3(14 \%)$ \\
Prior CABG & $0(0 \%)$ \\
Prior PCI & $5(24 \%)$ \\
Prior MI & $3(14 \%)$ \\
Diabetes & $4(19 \%)$ \\
Hypertension & $14(67 \%)$ \\
Hyperlipidemia & $12(57 \%)$ \\
Ml by LGE & $4(19 \%)$ \\
Beta-blockers & $12(60 \%)$ \\
Statins & $20(95 \%)$ \\
ACE-inhibitors/ARB & $9(43 \%)$ \\
Anti-coagulants & $17(81 \%)$ \\
\hline ACE angionsin
\end{tabular}

$A C E$ angiotensin converting enzyme, $A R B$ angiotensin receptor blocker, $C A B G$ coronary artery bypass grafting, $L G E$ late gadolinium enhancement, $M I$ myocardial infarction, $\mathrm{PCl}$ percutaneous coronary intervention coronary artery (LAD) of whom 3 underwent revascularization, 3 patients had stenosis in RCA of whom 2 underwent revascularization, 1 patient had stenosis in the left circumflex coronary artery (LCX) who underwent revascularization and 6 patients had no significant stenosis. Of the patients with CMR and PET 6 months after angiography 4 patients had stenosis in LAD of whom 3 had been revascularized, 2 had stenosis in RCA of whom 1 had been revascularized, 2 patients had stenosis in LCX of whom 1 had been revascularized and 2 patients had no significant stenosis. Complete records of the PET and CMR rate pressure products at both rest and stress were available for 12 patients. There were similar mean rate pressure products for PET and CMR both at rest $(8932 \pm 1563$ vs $9189 \pm 1756, p=0.37)$ and at stress $(10,945 \pm 2170$ vs $11,351 \pm 2463, p=0.53)$. Furthermore, the difference between stress and rest rate pressure products did not differ between PET and CMR $(2014 \pm 2554$ vs $2162 \pm 2120, p=0.75)$ indicating similar hemodynamic responses to adenosine stress.

\section{Global MP}

The global MP at rest and stress ranged from 0.6$4.0 \mathrm{ml} / \mathrm{min} / \mathrm{g}$ and $0.6-3.8 \mathrm{ml} / \mathrm{min} / \mathrm{g}$ for PET and CMR, respectively. Figure $2 \mathrm{a}$ shows that there was a strong correlation $(r=0.92 p<0.001 ; \mathrm{y}=0.94 \times+0.14)$ between MP quantified by PET and CMR. There was a good agreement between PET and CMR both at stress $(-0.1 \pm$ $0.5 \mathrm{ml} / \mathrm{min} / \mathrm{g})$ and at rest $(0 \pm 0.2 \mathrm{ml} / \mathrm{min} / \mathrm{g})$ (Fig. 2b). Furthermore, there was a significant correlation between global MPR by PET and CMR $(r=0.69, p=0.001 ; \mathrm{y}=$ $0.83 \times+0.53)$ and a good agreement $(-0.1 \pm 0.6 \mathrm{ml} / \mathrm{min} / \mathrm{g})$.

\section{Regional MP}

The regional MP at rest and stress ranged from 0.4$4.6 \mathrm{ml} / \mathrm{min} / \mathrm{g}$ and $0.5-5.3 \mathrm{ml} / \mathrm{min} / \mathrm{g}$ for $\mathrm{PET}$ and $\mathrm{CMR}$, respectively. Figure $3 \mathrm{a}$ shows the correlation between regional MP by PET and CMR $(r=0.83, p<$ $0.001 ; y=0.87 x+0.26)$. There was a good agreement between PET and CMR both at stress $(-0.1 \pm 0.7 \mathrm{ml} / \mathrm{min} / \mathrm{g})$ and at rest $(0 \pm 0.3 \mathrm{ml} / \mathrm{min} / \mathrm{g})$ (Fig. 3b). The correlation between MPR by PET and CMR was $r=0.57, p<0.001$ ( $\mathrm{y}=$ $0.68 \times+1.02)$ with a good agreement $(0 \pm 1.1 \mathrm{ml} / \mathrm{min} / \mathrm{g})$. Table 2 shows what the angiographer evaluated as significant stenosis by angiography in relation to regional stress $\mathrm{MP}<2.0 \mathrm{ml} / \mathrm{min} / \mathrm{g}$ as recently suggested as optimal cut-off for 13N-NH3 PET for detection of significant coronary stenosis [21].

\section{Global MP reduction}

Absolute quantification of MP is particularly important in cases exhibiting a global reduction in stress flow which arises in multi-vessel disease or microvascular disease. Figure 4 shows an example of a patient with a 


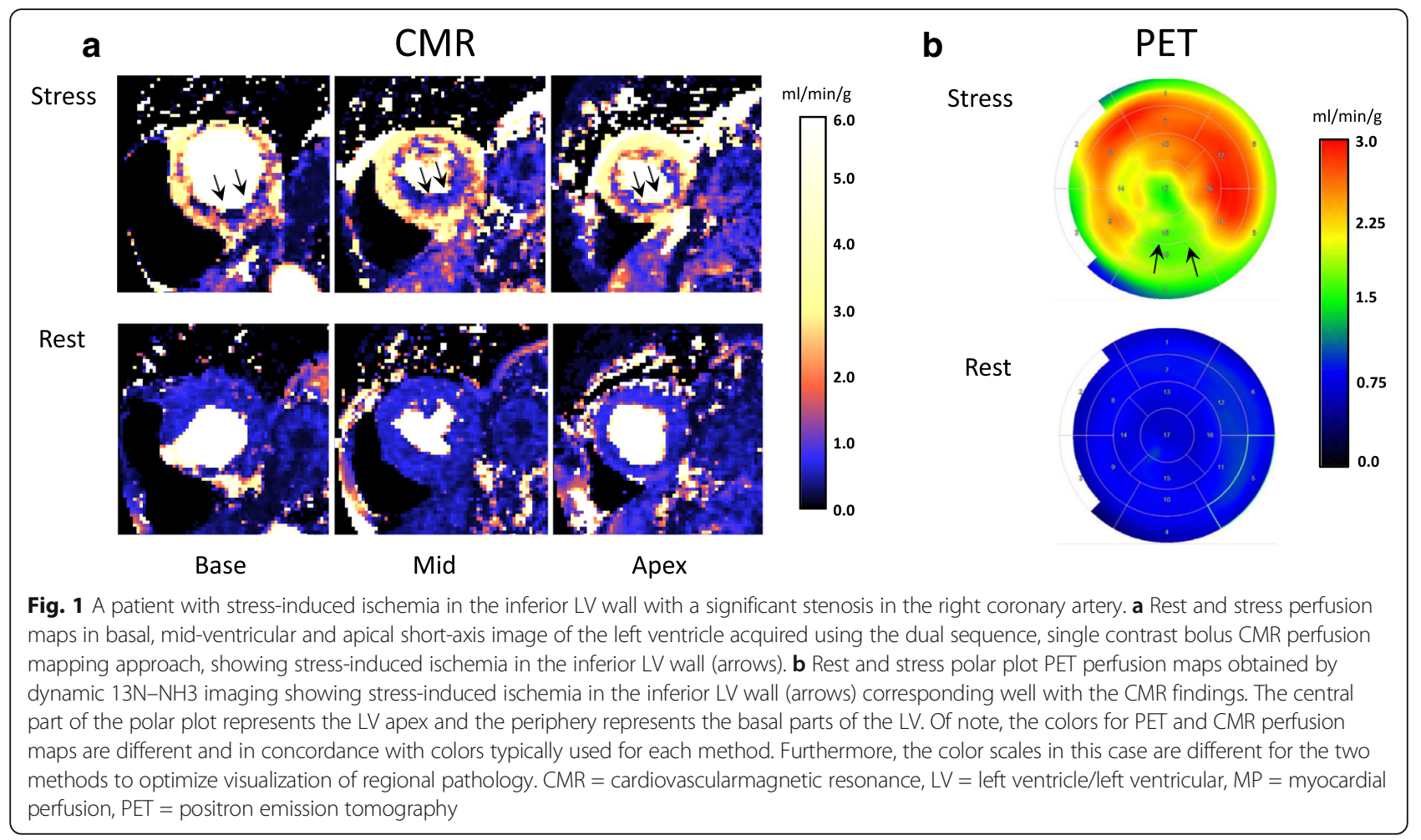

deceased global MP without regional ischemia on qualitative assessment of perfusion distribution. The MP appears to be normal in the raw first-pass CMR perfusion images (Fig. 4b) and in the non-dynamic, nonquantitative PET images (Fig. 4d), used in routine clinical practice for CMR and nuclear cardiology, respectively. Absolute quantification of MP (Fig. 4 a,c), however, clearly shows a significant reduction in flow and corresponding low flow reserve which could be attributed to either microvascular disease or multi-vessel disease. In this case, multi-vessel disease was confirmed by invasive angiography.

\section{Discussion}

The present study shows that fully quantitative CMR MP mapping is ready for clinical use by validating the
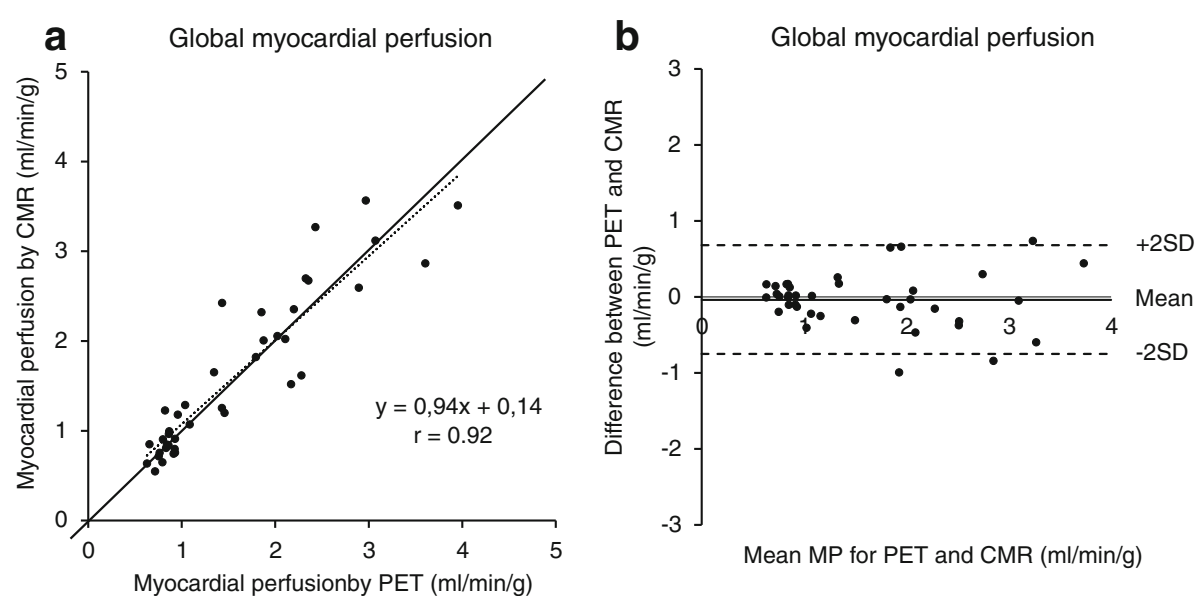

Fig. 2 The relationship between PET and CMR for global myocardial perfusion rest and stress included, a). The dashed line represents the line of regression. The solid line indicates the line of identity. $\mathbf{b}$ Bland-Altman plots for the difference between PET and CMR for global MP. The solid line represents the mean difference between the two methods and the dashed lines indicate +2SD and -2SD, respectively. There is strong correlation and small bias between PET and CMR for assessing global MP. CMR = cardiovascular magnetic resonance, MP = myocardial perfusion, $\mathrm{PET}=$ positron emission tomography 

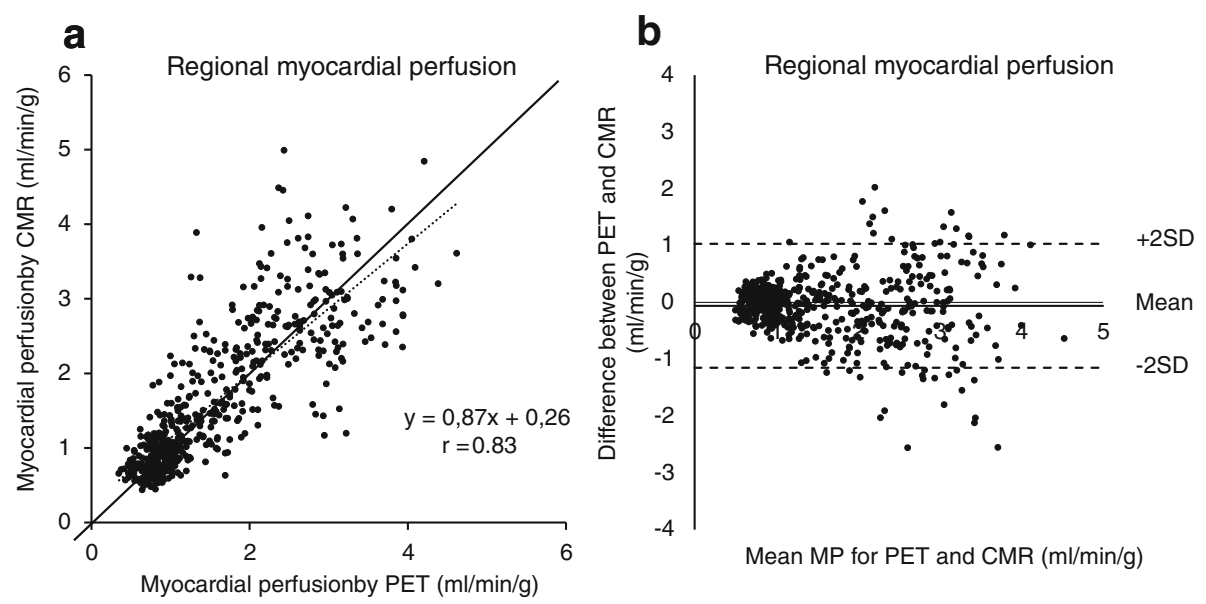

Fig. 3 The relationship between PET and CMR for regional myocardial perfusion (rest and stress included, a). The dashed line represents the line of regression. The solid line indicates the line of identity. $\mathbf{b}$ Bland-Altman plots for the difference between PET and CMR for regional MP. The solid line represents the mean difference between the two methods. The dashed lines indicate +2SD and -2SD, respectively. There is significant correlation and small bias between PET and CMR for assessing regional MP in 16 of the 17 segments of the left ventricle. CMR $=$ cardiovascular magnetic resonance, $\mathrm{MP}=$ myocardial perfusion, $\mathrm{PET}=$ positron emission tomography

method against 13N-NH3 PET, which was used as the independent reference standard. There was strong correlation between the two methods with negligible bias for both global and regional MP. Thus, using CMR for quantifying $\mathrm{MP}$ is an attractive alternative to PET in the clinic. However, routine clinical implementation of quantitative CMR MP will rely on its importance on therapeutic decision making, which needs to be proven by large clinical outcome trials that use $\mathrm{MP}$ as an endpoint.

Quantification of MP and MPR in routine clinical practice will have important implications on the management of patients with established or suspected CAD. Even though a normal perfusion scan with qualitative or semi-quantitative evaluation of first-pass perfusion has been shown to predict low risk of future coronary events [22-24], there remain patients in this group with adverse prognosis due to multi-vessel disease or non-obstructive CAD [25-27]. Thus, even in the absence of obstructive CAD, such as for 3 of 12 patients in Table 2, patients with pathologic MP at stress and low MPR are most likely in need of medical attention. The pathophysiological mechanisms explaining a decreased MP in the absence of obstructive coronary artery disease are likely

Table 2 Comparison between angiography and regional myocardial stress perfusion $<2.0 \mathrm{ml} / \mathrm{min} / \mathrm{g}$ for the 12 patients with PET (A) and CMR (B) before angiography

\begin{tabular}{lllllllll}
\hline A & & \multicolumn{2}{l}{ Angiography } & & B & & \multicolumn{2}{c}{ Angiography } \\
\cline { 3 - 4 } \cline { 7 - 8 } & & + & - & & & + & - \\
\hline PET & + & 6 & 3 & & CMR & + & 4 & 2 \\
& - & 0 & 3 & & & - & 2 & 4 \\
\hline
\end{tabular}

related to disease states shown to affect the coronary microvascular function, such as dyslipidemia [28, 29], hypertension [30], diabetes [31, 32], and smoking [33]. Significant vascular dysfunction related to these diseases may precede development of obstructive CAD [34], which makes quantification of MP and MPR potential diagnostic methods for identifying patients at risk of developing coronary stenosis. Furthermore, there are currently patients being treated with $\mathrm{PCI}$ in coronaries supplying myocardium with normal MPR [35]. Thus, these patients are put at risk associated with invasive treatment (endothelial injury, bleeding complications, risk of coronary artery dissection etc.) with little or no benefit to be gained from the procedure.

Using relative perfusion distribution, typically applied in routine clinical practice of nuclear cardiology, for diagnosing ischemic heart disease means that the counts in the perfusion maps are normalized to the highest regional counts. Thus, there might be variability in counts within normal myocardium due to variability of perfusion within the normal range. Hence, parts of the normal myocardium might have lower counts, potentially interpreted as ischemia and therefore compromising the diagnostic accuracy as recently shown by Kajander et al. [36]. They showed that the positive and negative predictive values for detecting or excluding invasively diagnosed fractional flow reserve (FFR)-positive coronary stenoses decreased significantly when using relative spatial perfusion distribution instead of quantitative MP.

One major challenge with quantitative perfusion imaging, including both CMR and PET in the present study, is to find a perfusion tracer with uptake kinetics that reflects the actual myocardial perfusion over a wide 


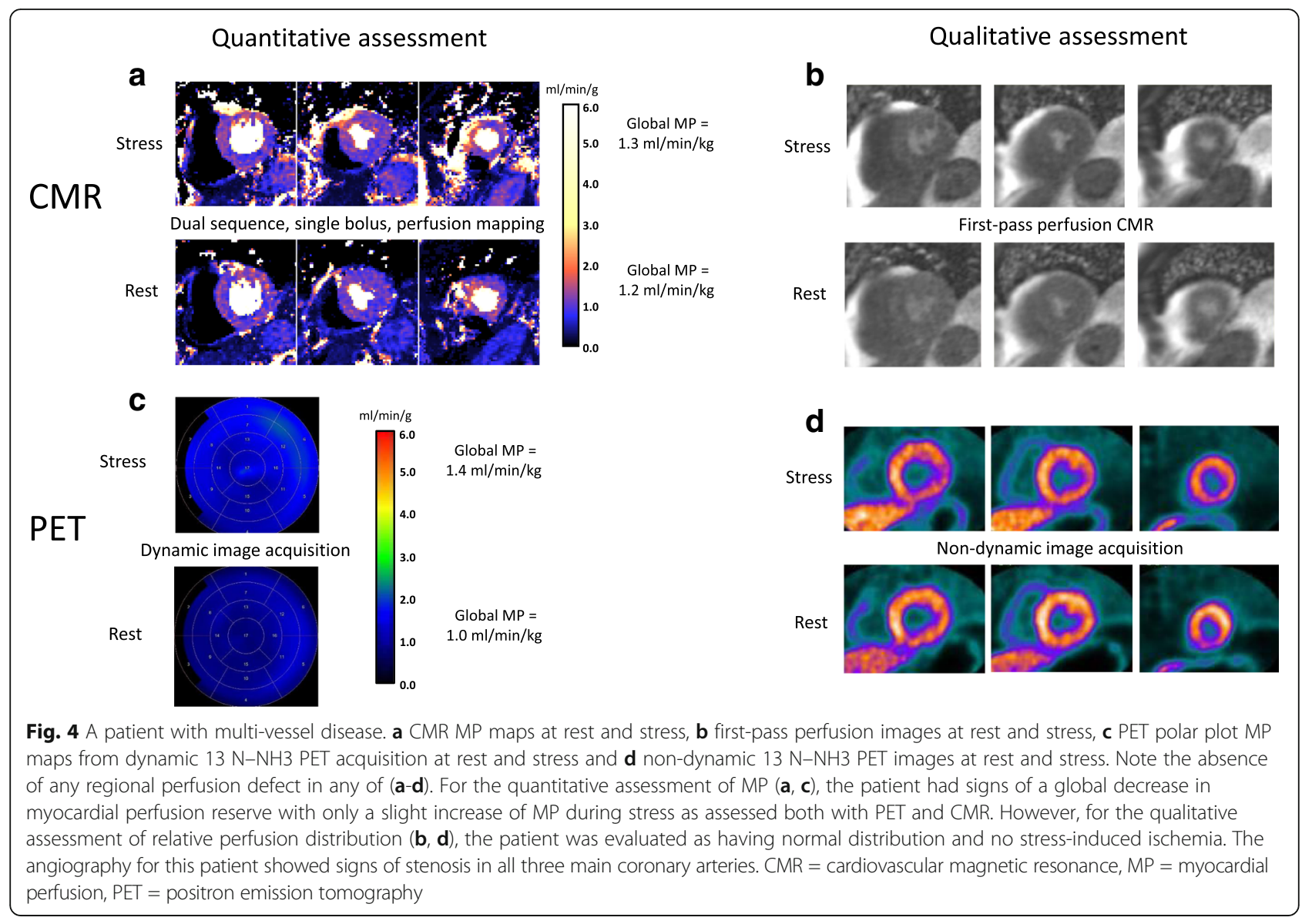

range of blood flows. It has previously been shown that $13 \mathrm{~N}-\mathrm{NH} 3$ uptake is approximately linearly proportional to flow at low MPs but that there is an underestimation of actual blood flow at high flows, which was compensated for by correcting for ammonia extraction [37]. The same underestimation has been shown for CMR and gadolinium-based extracellular contrast agents [38]. In the current approach [11], however, the gadolinium extraction fraction is estimated and used in calculating MP to avoid this underestimation of MP for CMR.

Imaging for both PET and CMR was initiated at the same time point $(3 \mathrm{~min})$ following the start of adenosine infusion. However, the duration for PET imaging and adenosine administration for the PET examination was significantly longer than for CMR. Thus, the adenosine response may differ somewhat [39] affecting the validation to some extent.

The results in the present study indicate that a dual sequence, single contrast bolus CMR perfusion imaging and $13 \mathrm{~N}-\mathrm{NH} 3$ PET have similar abilities in terms of quantifying myocardial blood flow over a wide range of flows. Thus, these techniques should be interchangeable and could therefore be used in accordance with local experience and expertise on the method of choice. A major limitation with $13 \mathrm{~N}-\mathrm{NH} 3 \mathrm{PET}$ is the cumbersome logistics with producing the tracer, requiring a cyclotron close to the imaging facility. Given the short half-life of $13 \mathrm{~N}-\mathrm{NH} 3(<10 \mathrm{~min})$ the tracer has to be produced and transported to the PET scanner within a limited time window. This requires advanced logistics and significant manpower, which also affects the cost per examination. With dual sequence, single contrast bolus CMR there are no such limitations. CMR offers superior characterization of myocardial morphology and function compared to $13 \mathrm{~N}-\mathrm{NH} 3 \mathrm{PET}$ due to its excellent spatial resolution. Furthermore, PET imaging is associated with ionizing radiation which CMR is not, making CMR the method of choice in younger patients and in situations where serial follow-up examinations is expected to be necessary, such as monitoring of treatment effects.

For wide spread clinical use of quantification of CMRbased MP, image acquisition, reconstruction and postprocessing need to be both automated and standardized. The CMR technique described in the present study is highly automated and standardized with regards to imaging protocol, image reconstruction and postprocessing of the data. The same requirements 
regarding automation and standardization is true for cardiac PET involving different perfusion tracers and different mathematical data modeling for quantification of MP built into different imaging software. The use of $13 \mathrm{~N}-\mathrm{NH} 3$ for quantification of MP and MPR has recently been shown to be stable across different software implementations [40].

Quantification requires knowledge of the amount of contrast agent in the myocardial tissue and the arterial input function (AIF) driving the delivery of this contrast agent. Accurate quantification is challenged by the lack of linearity between the measured signal and contrast agent concentration. The main sources of non-linearity and bias are: spatial signal variations caused by the sensitivity profiles of the surface coils, imperfect saturation of magnetization during contrast bolus passage, $\mathrm{T}^{*}$. decay (and signal loss) caused by high contrast agent concentrations in the blood pool, and the non-linear signal response inherent due to saturation recovery that depends on the parameters of the imaging protocol. The proposed dual sequence [11] is designed to address all of these issues. It has been proposed that some of the nonlinearity of the AIF response curve can be mitigated by imaging the AIF during a separate injection of a bolus with lower concentration (the dual bolus approach) [41], but this approach has some practical drawbacks as it requires multiple injections and a longer overall acquisition time. Moreover, there are other potential bias sources with this approach, since changes in breathing, etc. between the two measurements may introduce new sources of variation. Consequently, it is desirable to acquire the AIF curve simultaneously with the tissue response curve.

\section{Conclusions}

There is a good agreement between MP quantified by CMR with inline perfusion flow mapping and $13 \mathrm{~N}-\mathrm{NH} 3$ PET in patients with suspected stable CAD. Thus, CMR is a viable alternative in clinical practice for quantification of MP.

\footnotetext{
Abbreviations

ACE: Angiotensin converting enzyme; ARB: Angiotensin receptor blocker; CABG: Coronary artery bypass grafting; CAD: Coronary artery disease; CMR: Cardiovascular magnetic resonance; CT: Computed tomography; ECG: Electrocardiography; EWA: Expectation maximization, weighted intensity A priori information; FFR: Fractional flow reserve; IHD: Ischemic heart disease; LGE: Late gadolinium enhancement; LV: Left ventricle/left ventricular; MI: Myocardial infarction; MP: Myocardial perfusion; MPR: Myocardial perfusion reserve; OSEM: Ordered subsets expectation maximization; PCl: Percutaneous coronary intervention; PET: Positron emission tomography; PSF: Point spread function; SD: Standard deviation; SPECT: Single photon emission computed tomography; TOF: Time-of-flight
}

\section{Acknowledgements}

The authors acknowledge the technologists at the Department Clinical Physiology and Nuclear Imaging for their excellent skills and support in acquiring the CMR and PET data. Furthermore, the authors acknowledge Dr. Christos Xanthis for his engineering skills making the data acquisition possible.

\section{Funding}

This study was supported by Swedish Heart and Lund Foundation, Region of Scania, Lund University Medical Faculty.

\section{Availability of data and materials}

The datasets analyzed during the current study are not publicly available due to patient integrity but anonymized datasets are available from the corresponding author on reasonable request.

\section{Authors' contributions}

HE took part of designing the study, analyzing and interpreting all patient data and was a major contributor in writing the manuscript. HX took part of designing the study, processing of CMR raw data, generating CMR perfusion maps for analysis as well as for the manuscript illustrations and critically drafting the manuscript. SA was responsible for all data inclusion, analyzing the PET data and critically drafting the manuscript. MC took part of designing the study, interpreting data and critically drafting the manuscript. $\mathrm{CH}$ and $\mathrm{JO}$ was responsible for the technical aspects of the PET acquisition, participating in all PET data inclusion, participating in PET data analysis and critically drafting the manuscript. FH took part of the data inclusion, analysis of PET data and critically drafting the manuscript. MSH provided the technical foundation for all CMR data post-processing and critically drafting the manuscript. AA took part of designing the study, interpreting data and critically drafting the manuscript. PK took part of designing the study, post-processing of the CMR data, interpreting data and critically drafting the manuscript. HA took part of designing the study, interpreting data and critically drafting the manuscript. All authors have read and approved the final manuscript.

\section{Ethics approval and consent to participate}

The study was approved by the Lund University Regional ethics committee (\# 2013/550). All patients gave their written informed consent to participate in the study.

\section{Consent for publication}

Not applicable.

\section{Competing interests}

The authors declare that they have no competing interests.

\section{Publisher's Note}

Springer Nature remains neutral with regard to jurisdictional claims in published maps and institutional affiliations.

\section{Author details}

${ }^{1}$ Department of Clinical Physiology, Clinical Sciences, Lund University and Lund University Hospital, Lund, Sweden. ${ }^{2}$ National Heart, Lung, and Blood Institute, National Institutes of Health, DHHS, 10 Center Drive, Bethesda, MD 20892, USA. ${ }^{3}$ Department of Radiation Physics, Lund University Hospital, Lund, Sweden. ${ }^{4}$ Laboratory of Computing, Medical Informatics and Biomedical - Imaging Technologies, School of Medicine, Aristotle University of Thessaloniki, Thessaloniki, Greece.

Received: 24 May 2017 Accepted: 25 September 2017

Published online: 19 October 2017

\section{References}

1. Hachamovitch R, Berman DS, Shaw LJ, et al. Incremental prognostic value of myocardial perfusion single photon emission computed tomography for the prediction of cardiac death: differential stratification for risk of cardiac death and myocardial infarction. Circulation. 1998;97: 535-43.

2. Shaw $L$, Hachamovitch R, Berman DS, et al. The economic consequences of available diagnostic and prognostic strategies for the evaluation of stable angina patients: an observational assessment of the value of precatheterization ischemia. Economics of Noninvasive Diagnosis (END) Multicenter Study Group. J Am Coll Cardiol. 1999;33:661-9.

3. Ford ES, Ajani UA, Croft JB, et al. Explaining the decrease in U.S. deaths from coronary disease, 1980-2000. N Engl J Med. 2007;356:2388-98.

4. Roger VL, Go AS, Lloyd-Jones DM, et al. Heart disease and stroke statistics2012 update: a report from the American Heart Association. Circulation. 2012;125:e2-e220 
5. Smith SC Jr, Benjamin EJ, Bonow RO, et al. AHA/ACCF Secondary prevention and risk reduction therapy for patients with coronary and other atherosclerotic vascular disease: 2011 update: a guideline from the American Heart Association and American College of Cardiology Foundation. Circulation. 2011;124:2458-73.

6. Salerno M, Beller GA. Noninvasive assessment of myocardial perfusion. Circ Cardiovasc Imaging. 2009;2:412-24

7. Murthy $V L$, Naya M, Foster CR, et al. Improved cardiac risk assessment with noninvasive measures of coronary flow reserve. Circulation. 2011;124:2215-24

8. Herzog BA, Husmann L, Valenta I, et al. Long-term prognostic value of $13 \mathrm{~N}$ ammonia myocardial perfusion positron emission tomography added value of coronary flow reserve. J Am Coll Cardiol. 2009;54:150-6.

9. Tio RA, Dabeshlim A, Siebelink HM, et al. Comparison between the prognostic value of left ventricular function and myocardial perfusion reserve in patients with ischemic heart disease. J Nucl Med. 2009;50:214-9.

10. Gatehouse PD, Elkington AG, Ablitt NA, Yang GZ, Pennell DJ, Firmin DN. Accurate assessment of the arterial input function during high-dose myocardial perfusion cardiovascular magnetic resonance. J Magn Reson Imaging. 2004;20:39-45.

11. Kellman P, Hansen MS, Nielles-Vallespin S, et al. Myocardial perfusion cardiovascular magnetic resonance: optimized dual sequence and reconstruction for quantification. J Cardiovasc Magn Reson. 2017;19:43.

12. Hansen MS, Sorensen TS. Gadgetron: an open source framework for medical image reconstruction. Magn Reson Med. 2013;69:1768-76.

13. Bassingthwaighte JB, Wang CY, Chan IS. Blood-tissue exchange via transport and transformation by capillary endothelial cells. Circ Res. 1989;65:997-1020.

14. Heiberg E, Sjogren J, Ugander M, Carlsson M, Engblom H, Arheden H. Design and validation of Segment-freely available software for cardiovascular image analysis. BMC Med Imaging. 2010;10:1

15. Cerqueira MD, Weissman NJ, Dilsizian V, et al. Standardized myocardial segmentation and nomenclature for tomographic imaging of the heart: a statement for healthcare professionals from the Cardiac Imaging Committee of the Council on Clinical Cardiology of the American Heart Association. Circulation. 2002;105:539-42.

16. Engblom $H$, Tufvesson J, Jablonowski $R$, et al. A new automatic algorithm for quantification of myocardial infarction imaged by late gadolinium enhancement cardiovascular magnetic resonance: experimental validation and comparison to expert delineations in multi-center, multi-vendor patient data. J Cardiovasc Magn Reson. 2016;18:27.

17. Nesterov SV, Deshayes E, Sciagra R, et al. Quantification of myocardial blood flow in absolute terms using (82)Rb PET imaging: the RUBY-10 Study. JACC Cardiovasc Imaging. 2014;7:1119-27.

18. Nesterov SV, Settimo L, Mäki M et al. Imaging analysis systems—Carimas, FlowQuant and PMOD_-agree well on myocardial perfusion quantification of 13N-ammonia PET: a cross-comparison study. Abstract, EANM 2012, Milan, Italy 2012

19. DeGrado TR, Hanson MW, Turkington TG, et al. Estimation of myocardial blood flow for longitudinal studies with 13N-labeled ammonia and positron emission tomography. J Nucl Cardiol. 1996;3:494-507.

20. Bland JM, Altman DG. Statistical methods for assessing agreement between two methods of clinical measurement. Lancet. 1986;1:307-10.

21. Lee JM, Kim CH, Koo BK, et al. Integrated myocardial perfusion imaging diagnostics improve detection of functionally significant coronary artery stenosis by $13 \mathrm{~N}$-ammonia positron emission tomography. Circ Cardiovasc Imaging. 2016;9

22. Ingkanisorn WP, Kwong RY, Bohme NS, et al. Prognosis of negative adenosine stress magnetic resonance in patients presenting to an emergency department with chest pain. J Am Coll Cardiol. 2006;47:1427-32.

23. Lerakis S, McLean DS, Anadiotis AV, et al. Prognostic value of adenosine stress cardiovascular magnetic resonance in patients with low-risk chest pain. J Cardiovasc Magn Reson. 2009;11:37.

24. Pilz G, Eierle $S$, Heer $T$, et al. Negative predictive value of normal adenosinestress cardiac MRI in the assessment of coronary artery disease and correlation with semiquantitative perfusion analysis. J Magn Reson Imaging. 2010;32:615-21.

25. Uren NG, Crake T, Lefroy DC, de Silva R, Davies GJ, Maseri A. Reduced coronary vasodilator function in infarcted and normal myocardium after myocardial infarction. N Engl J Med. 1994;331:222-7.

26. Fujiwara $M$, Tamura $T$, Yoshida $K$, et al. Coronary flow reserve in angiographically normal coronary arteries with one-vessel coronary artery disease without traditional risk factors. Eur Heart J. 2001;22:479-87.
27. De Bruyne B, Hersbach F, Pijls NH, et al. Abnormal epicardial coronary resistance in patients with diffuse atherosclerosis but "Normal" coronary angiography. Circulation. 2001;104:2401-6.

28. Dayanikli F, Grambow D, Muzik O, Mosca L, Rubenfire M, Schwaiger M. Early detection of abnormal coronary flow reserve in asymptomatic men at high risk for coronary artery disease using positron emission tomography. Circulation. 1994;90:808-17.

29. Kaufmann PA, Gnecchi-Ruscone T, Schafers KP, Luscher TF, Camici PG. Low density lipoprotein cholesterol and coronary microvascular dysfunction in hypercholesterolemia. J Am Coll Cardiol. 2000;36:103-9.

30. Laine $\mathrm{H}$, Raitakari OT, Niinikoski $\mathrm{H}$, et al. Early impairment of coronary flow reserve in young men with borderline hypertension. J Am Coll Cardiol. 1998:32:147-53.

31. Di Carli MF, Afonso L, Campisi R, et al. Coronary vascular dysfunction in premenopausal women with diabetes mellitus. Am Heart J. 2002:144:711-8.

32. Yokoyama I, Momomura S, Ohtake T, et al. Reduced myocardial flow reserve in non-insulin-dependent diabetes mellitus. J Am Coll Cardiol. 1997;30:1472-7.

33. Campisi R, Czernin J, Schoder H, et al. Effects of long-term smoking on myocardial blood flow, coronary vasomotion, and vasodilator capacity. Circulation. 1998;98:119-25.

34. Zeiher AM, Drexler $\mathrm{H}$, Wollschlager $\mathrm{H}$, Just $\mathrm{H}$. Endothelial dysfunction of the coronary microvasculature is associated with coronary blood flow regulation in patients with early atherosclerosis. Circulation. 1991;84:1984-92.

35. Akil S, Hedeer F, Carlsson M, Arheden H, Hindorf C, Engblom H. Qualitative perfusion assessment by cardiac magnetic resonance imaging and invasive coronary angiography is not enough when evaluating patients with coronary artery disease - a cardiac positron emission tomography study. Abstract 2017;SCMR Scientific Sessions. Washington.

36. Kajander SA, Joutsiniemi E, Saraste M, et al. Clinical value of absolute quantification of myocardial perfusion with (15)O-water in coronary artery disease. Circ Cardiovasc Imaging. 2011:4:678-84.

37. Schelbert HR, Phelps ME, Huang SC, et al. N-13 ammonia as an indicator of myocardial blood flow. Circulation. 1981;63:1259-72.

38. Larsson HB, Fritz-Hansen T, Rostrup E, Sondergaard L, Ring P, Henriksen O. Myocardial perfusion modeling using MRI. Magn Reson Med. 1996:35:716-26.

39. Kitkungvan D, Lai D, Zhu H et al. Optimal adenosine stress for maximum stress perfusion, coronary flow reserve, and pixel distribution of coronary flow capacity by Kolmogorov-Smirnov analysis. Circ Cardiovasc Imaging 2017;10:e005650.

40. Slomka PJ, Alexanderson E, Jacome R, et al. Comparison of clinical tools for measurements of regional stress and rest myocardial blood flow assessed with 13N-ammonia PET/CT. J Nucl Med. 2012;53:171-81.

41. Christian TF, Aletras AH, Arai AE. Estimation of absolute myocardial blood flow during first-pass MR perfusion imaging using a dual-bolus injection technique: comparison to single-bolus injection method. J Magn Reson Imaging. 2008;27:1271-7.

\section{Submit your next manuscript to BioMed Central and we will help you at every step:}

- We accept pre-submission inquiries

- Our selector tool helps you to find the most relevant journal

- We provide round the clock customer support

- Convenient online submission

- Thorough peer review

- Inclusion in PubMed and all major indexing services

- Maximum visibility for your research

Submit your manuscript at www.biomedcentral.com/submit
C) Biomed Central 